\title{
"Goodbye, My Friencels": An Analysis of Incel Suicide Posts
}

\author{
Sarah E. Daly ${ }^{a}$ and Albina Laskovsov ${ }^{b}$
}

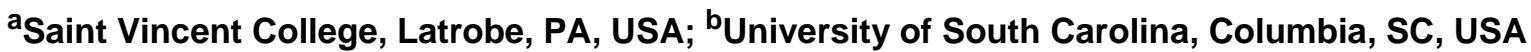

\section{ABSTRACT}

While an increasing number of researchers, non-profit organizations, and law enforcement agencies have expressed growing concern about the relationship between involuntary celibates (i.e., incels) and violence, no research to date has examined the potential for suicide and self-harm among men who call themselves incels. This study examines suicide posts shared to incel sub-Reddits that were collected by $u /$ IncelGraveyard $(N=80)$. The posts reveal that the notes discuss six subordinate themes, including the method of their planned attempt, the ways they used the internet, the online incel community, failed alternatives and coping, the effects of their suicide on others, and the afterlife. These broad themes offer a new and unique perspective about incels' experiences, perceptions, and coping strategies and add to the growing body of literature by highlighting the complexity of incel grievances. This study can inform future research and policy by encouraging gender-based and incel-specific treatment, reframing the incel discourse among researchers and mental health professionals, and arguing for a suicide prevention model to also prevent mass violence.
JQCJC "Qualitative

Criminology," (2022)

Vol. 11, Iss. 1, 1-33

ARTICLE HISTORY

Received 5/28/2021

Accepted 9/30/2021

\section{KEYWORDS}

Suicide; incels; mental health treatment; online community; suicide prevention

Incels, or involuntary celibates, have been the target of recent concern among media sources, law enforcement agencies, mental health practitioners, and non-profit organizations. In online spaces and specific forums, incels-defined as men who cannot achieve romantic or sexual success-often air their grievances, discussing their physical appearance, struggles with mental health, social critiques, and frustration with their situations (Ging, 2019; Glace et al., 2021; Menzie, 2020). Due to the often overtly violent, racist, or misogynistic nature of many of the posts, incels have often been associated with notions of mass violence, particularly as a result of violent attacks by alleged incels across the United States and Canada. This connection between violence and the entire incel community has led to sweeping generalizations about the group, and the Texas

DOI: https://doi.org/10.21428/88de04a1.b7b8b295

CONTACT Sarah E. Daly (sarah.daly@stvincent.edu), Saint Vincent College, Latrobe, PA, USA 
Department of Public Safety (2020) recently dubbed incels "an emerging domestic terrorism threat" (p. 3).

As incels become a growing area of study within a variety of disciplines including criminology and criminal justice, sociology, psychology, and more, academics have taken a variety of theoretical and methodological approaches to studying incel behavior and ideologies. While these have been informative and insightful, none to date have specifically examined the relationship between the incel identity, self-harm, and suicide. This study provides a brief overview of the incel literature followed by a synthesis of the existing research about tangentially related issues, including coping strategies, mental health treatment, suicide, and online communities. It then outlines the qualitative analysis of 80 suicide posts from incels that were collected by one Reddit user, u/IncelGraveyard, identifies emergent themes and subthemes, and offers recommendations for future research and policy. Though his methods for gathering these notes are unclear, they provide the most comprehensive collection of incel suicide notes available to date.

\section{Literature Review}

While incel research is a relatively new topic in many fields, the concept of frustration with one's romantic or sexual circumstances is not. While the concept of involuntarily celibate men has existed in literature throughout history and Gilmartin (1987) dubbed the situation "love shyness," the online manifestation of incels emerged in the late 1990s with Alana's Involuntary Celibacy Project. Shortly thereafter, Donnelly and colleagues (2001) examined involuntary celibacy as a concept, classifying respondents into three categories: virginal celibates, who had never partnered and had not had any sexual experiences; single celibates, who did not have partners but had sexual experiences; and partnered celibates, who were in a relationship and had past sexual experience but lacked sexual activity around the time of the survey. They found that respondents were people who "deeply desire a sexual relationship, who feel left behind by their age peers, and who are truly troubled by their lack of sexual intimacy" (Donnelly et al., 2001, p. 167).

Twenty years later, the notion of incels has morphed into a far more nefarious concept, with the online incel community often assumed to be interconnected with domestic terrorism, violence, and racism. Those who associate individual incels and the collective group with these ideas often point to examples of violence committed in the United States and Canada (see Hoffman \& Ware, 2020; Texas Department of Public Safety, 2020) in the name of incels or by those who espouse anti-woman sentiments, including the 2014 Isla Vista spree shooting (Serina, 2015); the 2018 Toronto van attack (Porter, 2021); the 2020 machete attack in a Canadian massage parlor (Bolsvert, 2020); and a New York City bomb threat by an alleged incel in April 2021 (Neumeister, 2021). 
While a comprehensive history of incels is beyond the scope of this article (for more information, see Daly \& Reed, 2021; Ging, 2019), it is necessary to understand the methodological approaches of prior criminological research about incels and involuntary celibacy. Most of the existing research has relied on content analyses of incels posts from sites like Reddit and incels.is (formerly incels.co) to identify common themes such as "the sexual market, women as naturally evil, legitimizing masculinity, male oppression, and violence" (O'Malley et al., 2020, p. 1) and "a constructed trope of women, the patriarchallyinformed male ideal, hypocritical approaches to gender, and the incel identity" (Maxwell et al., 2020, p. 1). Other researchers have examined the algorithmic nature of incel communities online (Papadamou et al., 2020), conducted large-scale linguistic analyses of the language of incel forums (Farrell et al., 2019; Heritage \& Koller, 2020; Tranchese \& Sugiura, 2021), and developed incel trait scales based on media reporting (Scaptura \& Boyle, 2020).

Much of this literature has attempted to unpack and measure incel beliefs, identities, and attitudes, but one of the most notable shortcomings of the existing research is its failure to fully examine the issue of suicide risk and related discussions among incels. Amidst the vitriolic rhetoric of incel forums, there often exist concerning discussions that reflect symptoms of severe depression, anxiety, and suicidal ideation. Even more, a 2020 survey conducted by incels.co shows that 74.1 percent of respondents reported that they "suffer from long-lasting anxiety, stress, or emotional distress," and 67.5 percent suffering from long-lasting depression (Anti-Defamation League [ADL], 2020).

Such data from the incel website is of concern given the relationship between suicide risk and co-morbid anxiety and depression in men (Bjerkeset et al., 2018). A prior 2019 incels.co survey indicated that 67.5 percent had considered suicide, further highlighting the increasing need to understand issues of suicide among incels (ADL, 2020). More information is necessary to examine the interactions between incel rhetoric, suicidal ideation, and depression, as the link between suicidality and mass violence cannot be ignored (Lankford, 2018; Peterson \& Densley, 2021). As Lankford (2013), explains, "At the bare minimum, there are three main requirements for suicide attacks: (1) suicidal intent (whether it's conventional, coerced, escapist, or indirect), (2) access to weapons, and (3) access to enemy targets" (p. 152). Additionally, for those who are considering suicide, there is the possibility of fame and notoriety (Lankford, 2016) for engaging mass violence and punishing others (either "taking someone with them" to death or making their death hurt survivors) while also aiming to die by suicide or law enforcement. Knowing this, it is imperative to consider the relationship between suicide and mass violence. 
Analysis of Suicide Notes

Olson and colleagues (2011) describe suicide notes as "unique sources of information written by a suicidal person ... [that] tell stories of emotional pain and cumulative despair" (p. 1492). Many people who attempt to die by suicide leave notes (Pestian et al., 2012), and prior research has identified trends among the content of suicide notes such as requests/mandates, methods, and expressions of emotions (Li et al., 2020), quantitative predictors of leaving suicide notes (Carpenter et al., 2016), and the presence of psychological difficulties such as aspiration or coping strains (Zhang et al., 2020).

Analyses of such notes can provide meaningful insight about particular issues related to suicide, and a qualitative approach can provide rich and meaningful analyses of the content of these artifacts, accurately reflecting the pain, hopelessness, and experiences of the notes' authors (for an exemplary exploration of analysis of suicide notes, see also Galasiński's (2017) Discourses of Men's Suicide Notes: A Qualitative Analysis). It is important to note that there should be a clear distinction between research regarding suicide notes from those who did, in fact, die by suicide, and those who may write them without the researcher knowing if they acted upon their ideations. Yet, the differences between online suicide notes, plans, or discussion and ones that are not posted on social media sites remain unclear. Liu et al. (2020) found that "[those who had attempted to die by suicide] demonstrated more suicide-related social media active use behaviors than [those who had suicidal] ideations but had not previously attempted to die by suicide" (p. 324).

Such research can provide insightful conclusions that can be of use to a variety of practitioners and researchers, as the "insights can enrich clinical care or strengthen existing suicide prevention programs" (Furqan et al., 2019, p. 98) and potentially prevent mass violence-suicide scenarios for vulnerable or high-risk populations like incels. Before examining suicide notes of incels, we begin with an overview of the literature related to suicide and this study in particular.

\section{Suicide}

Suicide rates have increased 35 percent from 1999 to 2018 (Hedegaard et al., 2020), but there still exists a gender gap between men and women in terms of lifetime suicide risk (Bachmann, 2018; Blair-West et al., 1999). Rasumussen et al. (2018) argued that young men transitioning to full adulthood are one of the highest risk groups in most countries, and Hedegaard et al. (2020) found that in 2018, "the suicide rate for males was 3.7 times the rate for females" (p. 3). Scholars have argued that means to engage in suicide behavior or social norms may explain why men's and women's suicide differs. 
One explanation for the gender disparity is the lethality theory, which highlights the differences in methods used. Tsirigotis et al. (2011) found that "female subjects tended to choose pharmacological drugs, overdose, and exsanguination as the suicide methods, while males more frequently used hanging and asphyxia" (p. PH65). Likewise, AdjacicGross et al. (2008) found that hanging was the most predominant method of suicide among males worldwide. The difference in the lethality of the method may also reflect the differences in the frequency of attempted suicide and completed suicides, as men tended to choose more violent deaths (Tsirigotis et al., 2011). As such, one would expect that incel suicides would utilize more violent methods.

Another explanation for the difference may be related to women's willingness to report nonfatal suicidal behavior and ideation. Rasmussen et al. (2018) found that "suicide in men was connected to feelings of inadequacy and shame generated by not meeting the culturally dominating gender role ideals" (p. 328), which is often the topic of many incel discussions. Because they fail to meet societal expectations of masculinity, incels often experience similar feelings of powerlessness and inferiority. Perhaps somewhat ironically, though, incels openly share these feelings and their romantic, social, and masculine failures, which is in stark contrast to the notion of male stoicism that is common among many cultures.

Despite incels sharing their negative experiences and emotions, they often express disdain at the notion of mental health treatment, as many believe it would fail to address the root causes of their inceldom (primarily their physical appearance). This may be linked to gendered notions of mental health treatment, the stigma of depression, and what is considered masculine in terms of behavior and even suicide. Krysinska (2014) posited that "in the United States and Western European countries, some means of suicide, such as hanging or guns (i.e., hard methods), might be considered more 'masculine' than the less lethal (i.e., soft) methods, such as self-poisoning, which are perceived as 'feminine'" (p. 15).

In a systematic review of the literature, Richardson et al. (2021) found 119 commonly identified risk factors, many of which can apply to incels, including lower education levels, being single, living alone, being of short stature or of a specific ethnic group, negative life events or trauma, and depression. The 2020 incels.co survey reflects many of these risk factors. Of the responses, 24 percent of incels claimed to be NEET (not in education, employment, or training), about 40 percent were of below-average height (between 5'0" and 5'8"), more than 50 percent only had a high school education, and nearly half reported that they were not white (ADL, 2020). 
Further, despite mixed findings of the relationship between loneliness and suicidal behavior, Gunn (2014) notes, "Some of the findings suggest a relationship, while others suggest that the relationship of loneliness to suicidal behavior was connected to its relationship to hopelessness, a well-documented risk factor for suicidal behavior" (p. 38). He continues by highlighting the negative effects of loneliness and hopelessness, particularly when comparing the outcomes to those of women who may experience similar feelings. This literature can also be beneficial for incel research when examining it in the context of Check et al.'s (1985) seminal work on the positive relationship between loneliness and aggression in men. Examining negative feelings due to isolation, friendlessness, and lack of relationships can provide a better understanding of adverse effects and outcomes.

Generally, the existing research can provide a useful framework for understanding how incel experiences - many which are rooted in issues of masculinity, inadequacy, and shame-can affect decisions related to suicide. As such, we apply these findings to incel suicide posts to better examine if and how incel behaviors are related to gendered understandings of self-harm, suicide, mental health treatment, and methods of death by suicide.

\section{Coping Strategies}

In addressing issues of depression, suicidal ideations, and other mental health challenges, research has evaluated coping activities and strategies and defined coping as "efforts to prevent or diminish threat, harm, and loss, or to reduce associated distress" (Carver \& Connor-Smith, 2010, p. 685). While many have classified and categorized different coping strategies, Felsten (1998) highlighted two fundamental types of coping strategies: problem-focused, which aims to actively "remove or reduce stressors through information seeking, planning, direct action, and seeking instrumental help," and emotionbased, which "involves dealing with emotional responses to stressors" (p. 290). He also recognized two proposed fundamental strategies including avoidance (such as distraction, denial, and social diversion) and seeking social support.

In addition to differentiating between problem- and emotion-focused coping, Carver and Connor-Smith (2010) similarly differentiate between other categories of strategies. They first describe engagement and disengagement strategies. Disengagement strategies (which are often emotion-focused) are similar to Felsten's (1998) avoidance strategies, while engagement strategies are problem-focused and aim to seek support and regulate emotions. Moreover, Carver and Connor-Smith (2010) describe accommodative coping and meaning-focused coping with the former focusing on acceptance, changing goals in the face of inevitable obstacles, and self-distraction. 
"Qualitative Criminology"

Meaning-focused coping, coined by Folkman (1997), aims to find benefit or value from stressful experiences.

More recently, in a qualitative investigation of men's positive strategies for managing depression, Fogarty and colleagues (2015) found that many men "reported the successful use of a broad range of positive strategies" such as physical activity, enjoyable activities, engaging in therapy, and developing a plan for self-care (p. 185). However, in a systematic review of the masculinity and coping literature, Spendelow and Seidler (2020) found that men also can use flexible masculinity to challenge existing masculinity traits or, conversely, use existing masculinity (and resisting change) to "characterize or perform tasks/behaviors, maintain control over one's circumstances, exhibit traditional/hegemonic attributes, and resist individual and/or lifestyle changes in the face of a life challenge" (p. 108). They, too, noted positive adjustment and avoidance as superordinate themes in the literature.

We apply this area of research to frame the ways individuals use distractions or engagement to stave off negative feelings and suicidal ideas. The literature provides an understanding of the types of strategies that could be used for self-care and the ways that masculinity can affect coping mechanisms. This study not only examines the types of coping mechanisms that incels used, but also highlights the ways in which they were insufficient in preventing suicidal plans.

\section{Mental Health Treatment}

Mental health treatment for suicidal ideation, depression, and other mental illnesses is an effective tool for reducing and preventing suicide attempts. Treatment may include inpatient or outpatient services in which various therapies and pharmaceutical approaches may be utilized. Research has shown that mental health treatment does, in fact, reduce the risk of suicide (Katz et al., 2013; McClellan et al., 2021), and it has evidenced that mental health treatment utilization is significantly lower in individuals with depressive symptoms and suicidal ideation (Deane et al., 2001; Hom et al., 2015). Gibbons and colleagues (2010) found that individuals who drop out of mental health treatment programs or do not complete the program report doing so because of greater life stressors, high anxiety, depressive symptoms, and overall poor coping skills. Other challenges to accessing mental health treatment services include housing and income instability, negative past experiences with treatment centers and specialists, a sense of hopelessness, and the fear associated with disclosing mental health concerns (Alonzo et al., 2017).

Mental health treatment may work as a protective barrier for suicide and so understanding the accessibility and availability of various mental health services is 
important in suicide prevention (McClellan et al., 2021). Those individuals with the highest risk for suicide (i.e., those with suicidal ideation or a history of suicide attempts) are the most difficult to include in various mental health treatment centers and services, as this population is often prone to early program termination (McClellan et al., 2021). However, increases in outpatient service staffing, increased funding, widely implemented resources, and greater accessibility have the potential to alleviate the pressures of ineffective mental health treatment (Katz et al., 2013). It is also significant to consider that many individuals with suicidal ideation are more readily willing to seek support for people in their immediate support system, including family members and friends (Deane et al., 2001). Reliance on informal social supports rather than formal, professional services can mediate suicidal ideation and aid in the prevention of further suicide attempts.

By examining the impact of mental health services on individuals with mental health concerns, like suicidal ideation, we apply this knowledge to incels who experience suicidal ideations. The current study points to the lack of access to mental health services and an over-reliance on informal social support systems, like online chatrooms, which allow for the sharing of incels' experiences and their various mental health concerns. When considering potential policy implications for suicide prevention of this vulnerable population, we use this body of literature to inform our assessment of incels' experiences with suicidal ideation and how they navigate help-seeking services.

\section{Online Communities and Pro-Suicide Forums}

One example of informal social supports comes from online communities, particularly those that offer emotional assistance and positive affirmations (Recupero et al., 2008). The internet provides the space for online communities to coalesce and interact, often through forums, online chat rooms, social media platforms, and other private digital enclaves. Incels' navigation of the internet includes using various digital spaces for helpseeking, social support, and participating in discourse surrounding mental health and suicide ideation. Of particular importance to the current study is understanding what role the internet plays in promoting pro-suicide sentiments while also providing a community of support and help-seeking. The internet provides a variety of information, such as where to find help, access mental health resources like clinics, therapists, and pharmacies, and positive peer encouragement. Additionally, informal support groups may be accessed through digital means (Recupero et al., 2008). On the other hand, the internet can be accessed to view pro-suicide forums. These forums typically provide information about suicide methods, resources for accessing the means to die by suicide, facilitate suicide pacts, and use peer pressure and coercion to encourage others to suicide (Biddle et al., 2008). Accessibility and availability of suicide-related websites and online resources are 
"Qualitative Criminology"

known to affect the likelihood of a vulnerable individual attempting suicide (Recupero et al., 2008).

Research indicates that the internet plays a vital role in suicidal ideation and community fostering of both pro- and anti-suicide sentiments. For instance, one study about online users of suicide-related digital media and platforms found that those who sought online resources about suicide reported higher levels of social anxiety as well as lifetime suicidal ideation (Mok et al., 2016). Alternatively, Biddle et al. (2008) found that pro-suicide websites are widely accessible, and therefore may aid in the completion of suicide attempts of people visiting such sites. Online communities' proliferation of various suicide-related sentiments within digital spaces has the potential to influence an individual's decision regarding suicide.

\section{Post-Suicide Concerns}

Some who write suicide notes express concerns or projections for what would happen to them. In terms of post-suicide considerations or trepidation, they may write about the effects or aftermath of their suicide. They may discuss their expectations for or mentions of the afterlife or share concern for others they are leaving behind. Li et al. (2020) categorized post-suicide concerns as mandates or requests including disposal of possessions, blame, and care of family as well as expressions in which the author of notes asked for forgiveness or discussed expectations or blessings. For "suicide survivors," or those left behind, the tragedy of the death of a loved one may be related to the traumatic aftermath, stigma, shame, isolation, mixed emotions, and a need for a reason (Harvard Health Medical School, 2019). As such, those who are contemplating suicide may consider how their actions will affect those who may be affected by their death.

As it pertains to suicide and the afterlife, Gwim et al. (2020) point out that "many [religious] denominations prohibit suicide, which may contribute to the associations between religiosity and suicide" (p. 258). Similarly, many studies have found religiosity to be a protective factor for suicide (Koenig, 2008; Moreira-Almeida et al., 2006). Yet, in the United States, religiosity and the religious landscape are changing, with 26 percent of adults "describ[ing] their religious identity as atheist, agnostic or 'nothing in particular' ... up from 17\% in 2009" (Pew Research Center, 2019, para. 1). Regardless of religious affiliations, a National Post survey found that while 66 percent of all respondents believed in life after death, 70 percent of the Millennials in the study (aged 18-29) reported that they did, exceeding the rates among Generation X respondents, Boomers, and PreBoomers (Brean, 2018).

Likewise, Li et al. (2020) found that "25.1 percent of [suicide notes] mentioned life after death or beliefs about death" (p. 36) with notions that they would bless their family 
members, lived a cursed afterlife, or enter a world for dead people. These results were from a study of suicide notes in China, so they may differ from western or Euro-centric beliefs about the afterlife such as heaven and hell. Comparisons of suicide notes left behind by Native American, Hispanic, and Anglo people found that more than 20 percent of notes referenced reunification or notions of the afterlife (Olson et al., 2011).

Given that 67.4 percent of respondents in the March 2020 incels.co survey reported being Atheist/Agnostic, this study aims to understand the role that religion or the afterlife may play in incels' decisions and plans to die by suicide. Even more, given the relatively solitary, lonely nature of the prototypical incel, we looked to the literature to understand and frame the ways that concern for others may affect their decisions to die by suicide, echoing the finding of Maxwell et al. (2020), "This deep level of social isolation led to many members of the incel community expressing thoughts of suicide and wondering if anyone would miss them if they were to commit suicide" (p. 1864).

\section{Current Study}

$\mathrm{u} /$ IncelGraveyard is a Reddit user (whose true identity is unknown) who compiled a list of incel suicide posts. As of January 20, 2021, there were 94 links to screenshots of Reddit posts, all from unique users. Of the 94 cases, we dropped 12 because links to messages no longer worked, one because it was a message about a friend's suicide (and not a direct post from a person considering suicide), and one because it was not written by an incel (by most traditional definitions). This left us with 80 total suicide posts for this analysis, and they ranged from 7 words ("I'm out. Thanks for the blackpills. Goodbye.") to 1,186 words, with an average of 165 words per note. Descriptive statistics about the authors of the posts are largely unavailable, as only a few offered demographic information about race, ethnicity, country of origin, or age. The 2020 incels.co survey offers a bit more insight about incels, but we cannot assume that the data from that website's survey would be generalizable to the Reddit incel population. However, we can be almost certain that the vast majority of incels whose posts were shared on this Reddit user's site are male, as many incels are of the belief that women cannot be incels.

As noted in the limitations section of this paper, there is no way to know if the authors of these notes died by suicide. While some comments may provide some insight about the outcomes for the users (e.g., one now deleted account wrote, "Miss him. He was very close to me"), this does not necessarily provide confirmation that he died. There is still value in these notes, though, as they offer the opportunity to examine incel perspectives, and, in particular, information about their experiences, plans to die by suicide, and attempts to cope. 
"Qualitative Criminology"

\section{Method}

We utilized the Braun and Clarke's (2006) six phases of thematic analysis, following the 15-point checklist. As Chatfield (2018) notes the limitation of the passive term "themes emerged," we instead point out that we coded the text, actively engaging with the data, and used systematic ways to identify the themes. Initially, we visited the u/IncelGraveyard page and compiled all the screenshots and posts into a single document, numbering them and noting those with non-working links. We then transcribed the screenshots to text in the document, including photos that were included in the posts. We immersed ourselves independently in the data, reading through several times and familiarizing ourselves with the content, noting initial ideas. Then, we began "coding interesting features of the data in a systematic fashion," (Braun \& Clarke, 2006, p. 87), and we then met to discuss. We collated codes into potential themes, and ultimately decided upon the six major themes and conceptualized them.

Next, we evaluated the text for these themes using Microsoft Word's comment feature to highlight the text using non-discrete coding with multiple themes often appearing in single posts. We met periodically to discuss these, making adjustments to the definitions for each and reviewing the data and recoding. Finally, we coded again for the final themes and calculated the number of notes in which they appeared. Although we included the frequency of the themes across notes, we recognize that the importance of a theme is not dependent on the quantity of its appearance across the data. We did, however, want to provide at least a basic determination of the frequency of the themes across users to capture the shared experiences of the suicidal incel identity. Upon completion of this, we began the process again to identify subthemes, finalized the codebook, and ultimately discussed, conceptualized, and coded again.

Once we had each finalized our code for the themes and subthemes in our own independent documents, we merged the documents and used macros developed by Fredborg (2013) to compile a table of comments. As utilized in previous research, we calculated the inter-rater reliability (IRR) using the number of agreements over the total number of codes (McAlister et al., 2017; Miles \& Huberman, 1994). In total, we had 89 percent agreement across the 80 posts. We also calculated the IRR for each of the themes, which ranged from 86 percent to 100 percent agreement.

\section{Positionality of the Research Team}

It is important to note our personal perspectives and identities as they may relate to our coding and understanding of incel issues (McCorkel \& Meyers, 2003; Naples, 2003). We are both cisgender, heterosexual women, and these identities may affect the way that we viewed some of the content in the posts, and in particular, those which denigrated women. 
While we do not condone or support the most radical of incel beliefs as they often appear on forums, we recognize that incels must be humanized and understood to effectively prevent and address the likelihood of violence or, in a seemingly more likely outcome, suicide and self-harm. We understand that it is difficult to view incels in a sympathetic light, particularly given the heinous nature (e.g., violence, misogyny, racism, etc.) of many forum discussions, and we discussed this throughout the research process. Ultimately, though, we believe that the findings from this study lend themselves to prevention and intervention practices and efforts to address issues of suicidality and violence.

Additionally, given our positionality, the authors took care to engage in reflective practices and check in with each other given the sensitive and depressing nature of the content we analyzed. During the meetings in which we discussed themes and coding, we often debriefed about specific posts or shared the ways that we would use self-care practices (e.g., watching television/movies, spending time with friends, stepping away to work on other projects). The collaborative nature of this project allowed us to help each other address our own personal feelings about the suicide notes.

\section{Results}

This section discusses the major themes (and related subthemes) that emerged from the data: method of suicide, the function of the internet, the incel community, attempted alternatives or coping, the effects for others, and the afterlife. The six primary themes are listed in Table 1 with the name, the definition or conceptualization of the theme, a representative quote, and the IRR calculation for each theme. For each theme (except the afterlife), subthemes are described with relevant quotes.

\section{Method of Suicide}

Seventy percent $(n=56)$ of the posts mention the methods that the individuals planned to use to complete their suicide. While many used the term "rope," a denominalization used among incel forums implying to die by suicide, others were more specific about the means they wanted to use.

\section{Metonymic "Rope"}

On incel sites and on Reddit threads, the term "rope" is often used as a verb (e.g., "to rope") or as a noun (e.g., "the rope is calling") indicating their desire to die by suicide. This metonymic reference which associates the means to end one's life and the act also, at times, indicates that the authors felt "called" to consider suicide (when used as a noun, while others simply chose to use it to indicate the action of dying by suicide). Approximately 44 percent $(n=30)$ of posts included this metonymic rope, although some 
stated that they were planning to rope and then listed a non-hanging method of suicide. One user concisely noted, "I'm roping tonight" (Post 4), while another stated, "I'm going to fucking rope" (Post 55).

Table 1. Suicide Post Themes

\begin{tabular}{|c|c|c|c|}
\hline Primary Theme $(\mathrm{n})$ & IRR\% & Definition & Representative Quote \\
\hline Method $^{*}(n=56)$ & $100 \%$ & $\begin{array}{l}\text { The means or plans to } \\
\text { attempt suicide or a } \\
\text { metonymic mention of } \\
\text { "roping" }\end{array}$ & $\begin{array}{l}\text { "I have bought a shotgun with } \\
\text { my financial aid money when } \\
\text { my college started." }\end{array}$ \\
\hline The internet* $(n=11)$ & $91 \%$ & $\begin{array}{l}\text { Use of the internet for non- } \\
\text { forum purposes }\end{array}$ & $\begin{array}{l}\text { "After } 24 \text { years of constant } \\
\text { suffering [I] finally ordered } \\
\text { potassium cyanide pills from } \\
\text { the net." }\end{array}$ \\
\hline community* & $86 \%$ & $\begin{array}{l}\text { Directly addressing people } \\
\text { on the forums or incel } \\
\text { group; function of the } \\
\text { community }\end{array}$ & $\begin{array}{l}\text { "I appreciate all the people } \\
\text { here, who actually } \\
\text { understand what it's like to be } \\
\text { a genetic defect ..." }\end{array}$ \\
\hline Alternatives ${ }^{\star}(n=53)$ & $88 \%$ & $\begin{array}{l}\text { Means of coping or } \\
\text { attempting to improve their } \\
\text { situation or mental health } \\
\text { conditions }\end{array}$ & $\begin{array}{l}\text { "I tried everything, playing an } \\
\text { instrument, working out, new } \\
\text { clothes, new styles, new hair } \\
\text { cuts, whatever the fuck. None } \\
\text { of that shit matters." }\end{array}$ \\
\hline $\begin{array}{l}\text { Effects for others* } \\
(n=16)\end{array}$ & $94 \%$ & $\begin{array}{l}\text { Concern or revenge for } \\
\text { those who may be affected } \\
\text { by their death }\end{array}$ & $\begin{array}{l}\text { "I just hope my mom won't be } \\
\text { devastated when she hears } \\
\text { about me." }\end{array}$ \\
\hline The afterlife $(n=20)$ & $95 \%$ & $\begin{array}{l}\text { Reference to some life } \\
\text { after death }\end{array}$ & $\begin{array}{l}\text { "hope to see you all in a } \\
\text { better place." }\end{array}$ \\
\hline
\end{tabular}

*Indicate a primary theme that includes sub-themes.

\section{Ingestion}

Eleven percent of posts $(n=8)$ reference ingestion as a means of suicide. Of particular note is the use of chemicals to complete their suicide. One user wrote, "I have a syringe full of sodium thiopental ready beside me as I type this" (Post 67). While the post implies the lethality of the drug, it is still important to note that if used in inappropriate quantities, sodium thiopental, a barbiturate used in the lethal injection process, can lead to a severe drop in blood pressure, respiratory difficulties, and shock (rxlist.com, 2018), thus indicating a seemingly painful death. Another user shared, "The Potassium Dichromate mixture I drank an hour ago will have me dead shortly" (Post 40). Several medical journals 
describe an overdose of this chemical, which is often used in cleaning materials or artistic/photographic chemicals, resulting in liver and renal toxicity (Illner et al., 2009; Kołaciński et al., 1999). Another user, however, used a less complex approach to ingestion, writing "I think the rat poison is starting to work" (Post 26).

\section{Jumping}

In 8.6 percent of posts $(n=6)$, the users planned to either jump from a high place or in front of a train as their means of suicide. One user wrote, "I'm going to go and jump off a bridge today" (Post 38), and another wrote, "I am jumping off a high rise" (Post 28). One user wrote, "I'm on my way to kill myself by jumping in front of a train" (Post 16).

\section{Suffocation/Hanging}

While 44 percent of the authors utilized the metonymic "rope" as a noun or verb to describe death by suicide, 8.6 percent of the total users $(n=6)$ specifically mentioned suffocation or hanging as their means of death. Post 63 cited a specific website on which he found the exit bag method, which involves running a pipe from a gas container into a bag around his head. Another post explained, "I ordered a helium setup a year ago when I still had my job at Kroger" (Post 84), which can be used in the same way as the exit bag method (Smędra et al., 2015). Yet another user wrote, "Any interested suicidecel can research partial suspension hanging" (Post 94). This approach differs from the previous "rope" subtheme, as it provides a specific method of suffocation or hanging, while the earlier use of the term is a more generic term indicating the desire to die.

\section{Gun}

Despite the existing literature speculating that death by weapons may be more masculine, only 5.7 percent of posts $(n=4)$ expressed an explicit intention to use a gun as their primary means. One user associated the gun and/or the planned suicide with masculinity, writing, "I maned [sic] up and bought a shotgun today" (Post 28). Another user shared, "I have bought a shotgun with my financial aid money when my college started" (Post 15). While many would suspect that men would choose firearms as the most common means of male suicide, it is important to note that members of the incel community exist across the world (not simply the United States), and access to guns may be limited.

\section{The Function of the Internet}

The internet is an important element of the incel experience, notably because this community operates within the confines of the digital space. It is then unsurprising to 
"Qualitative Criminology"

discover that the internet played an important role for incels posting on the $\mathrm{u} /$ IncelGraveyard page. Findings revealed mentions of another sub-Reddit, called $r /$ IncelTears $(n=5)$, which operates as a gag account that essentially trivializes and denigrates the incel experience. Additionally, the internet played a vital role in providing the means through which suicide methods were researched and subsequently used to then purchase the items necessary to complete suicide $(n=6)$.

\section{Researching/Purchasing Methods}

Researching suicide methods and purchasing means through which to accomplish suicide is not unique to the incel community, but rather serves as an extension to this online community. For instance, one incel posted, "I have purchased cyanide from the darknet ..." (Post 1) and another posted, "After extensive research, I have chosen the Exit bag method" (Post 63), providing a link to a website that explains the process he planned to use. These quotes reveal an accessibility to both the information about suicide methods as well as an accessibility to the means to accomplish suicide. In further discussions regarding suicide prevention, this accessibility should be considered as a potential mediating point.

\section{IncelTears}

It is established in the current study that $r /$ IncelTears operates as a bullying and demonizing outlet on the internet. This sub-Reddit primarily utilizes comedic and humorous tactics of disparaging incels by providing sarcastically ironic commentary about incel posts on other incel forums (Dynel, 2020). IncelTears identifies as a "part-mocking, part-watchdog" (Dynel, 2020, p. 2) sub-Reddit and it is this disparaging that incel users found most problematic and discouraging. As one user posted "constant harrassing [sic] from IncelTears users has not helped at all" (Post 19), and another wrote, "I want to say despite how much IT [IncelTears] demonizes this place while saying people here use suicide encouragement, I have never been more drawn to suicide than I have arguing with them" (Post 84). These sentiments evidence the effect that bullying (exacted by other online groups such as IncelTears) has on incels, particularly those who are vulnerable to suicidal ideation as determined by coexisting depressive symptoms.

\section{The Incel Community}

An overwhelmingly prominent finding in the current study is that of a sense of community, wherein at least 95 percent of the users made a reference to the online incel community $(n=76)$. The online forum provided a place for many incels to seek friendships and companionships that provided connections and solidarity, particularly through their 
shared lived experience of identifying as incels. There was often a unifying solidarity around being ostracized by society and marginalized by "normies" (slang for the "normal" people who do not understand incels' challenges and struggles), highlighting the ways in which they navigated normativities (Coimbra-Gomes \& Motschenbaher, 2019; Heritage $\&$ Koller, 2020). Users on the forum identified various life stressors and anxieties that often led to the decision to plan their suicide, while many of the posts also revealed gratitude for the community, and it was this online community of like-minded individuals that provided relief, albeit temporary, from those life stressors and anxieties. Findings revealed a distinction within the community posts in which the majority of users who directly commented about the incel community (88\%) provided positive encouragement and only rarely did they provide negative encouragement (8\%).

\section{Positive Encouragement}

Positive interactions were identified as those interactions in which users thanked the incel community as well as individual incels for their friendship and their support $(n=67)$. This is evidenced in the following quote, "I felt like I was welcomed in this community. For that, I want to thank each and every one on here for allowing me to enjoy myself and enjoy life for a little bit longer" (Post 42). Positive interactions made up the majority of the 76 total posts for this theme and this reveals the important relationship that incels have with one another and the larger online community.

\section{Negative Encouragement}

Alternatively, negative interactions made up those instances wherein the post was negative in some way, either through denigration of the incel community or encouragement for other incels to die by suicide $(n=6)$. This sentiment is present in the following quote, "I would actively encourage people like us to just fucking end it" (Post 81). Negative encouragement for other incels to subsequently die by suicide was often shrouded in one's own sense of hopelessness and lack of alternative options. Although only six posts provided negative encouragement, this finding is an important one in understanding the diverse reactions and complex relationships within the incel community.

\section{Attempted Alternatives or Coping}

Alternatives to dying by suicide is a prominent theme in the current study. This reflects the decision-making process for many incels from seeking means of distraction (an avoidant or accommodating coping mechanism), as well as considering mental health treatment before the decision to plan their suicide. While many "normies" simply suggest 
that incels utilize escorts or sex workers (see Costello, 2020), only one user mentioned this as an attempt to cope with his situation. Instead, the vast majority of posts indicated a variety of techniques to cope with their situation or negative feelings.

\section{Mental Health Treatment}

In understanding the trajectory of some incels' decision to take their life, some users suggested that they had attempted to secure mental health treatment $(n=9)$. The posts also revealed the ineffective and insufficient access and availability to such services. For instance, one user posted "the mental hoslatpl [sic] is worse than death" (Post 28), and another revealed that "I tried to phone the doctors, and suicide hotlines, but the doctors don't pick up, and then suicide hotlines just give me platitudes" (Post 44). These quotes reflect the regular unavailability of mental health services, which affects the decisionmaking process towards suicide.

\section{Coping/Distractions}

Furthermore, alternatives to dying by suicide were discussed by incels as coping mechanisms (or copes) and distractions $(n=44)$. One user posted "I have also run out of copes, music, movies, alcohol, drugs, video games, anime, porn, religion, you name it" (Post 63). These coping activities included various hobbies like media consumption, video gaming, ingestion of alcohol and drugs, and participation in religious and spiritual activities. Coping tactics were mentioned in the majority of posts as a clear escalation from distracting activities to considering suicide and the subsequent decision to complete suicide. Coping mechanisms were then subdivided into various practices and tactics.

\section{School/Jobs}

Eleven posts referenced having a job or career or being in school as a way to cope with their situation. These copes were often disparaged by incel users as having a job, even a good job, did not prevent the consideration to dying by suicide. As one user posted "It fucking sucks barely making it in and having several tens of thousands of dollars of debt waiting for you in a few years" (Post 55) and another rhetorically asks "what's the point of being a great A [sic] law student when your psyche is fucked up and shit" (Post 80). Having a job or being in school does not preclude one from considering and attempting suicide. 
Improving Physical Appearance

Ten posts referenced attempts to improve their physical appearance through looksmaxxing or gymmaxxing. Looksmaxxing $(n=8)$ is uniquely incel terminology in which young men are encouraged to participate in a beautification process. Considering the significant emphasis on one's physical appearance within the incel community, looksmaxxing provides the means of changing one's appearance as an attempt to attract a potential romantic partner. Looksmaxxing takes on a variety of practices such as changing one's hair style, tanning, teeth whitening, acne treatment, and altering clothing styles. One user stated, "I've tried me [sic] hardest and poured my heart and soul into improving my appearance ... " (Post 79) and another declares "I will never be able to ascend no matter how hard I looksmaxx" (Post 57). For many incels, looksmaxxing is not a sufficient cope and often does not provide the relief they seek.

In addition to looksmaxxing, gymmaxxing $(n=2)$ is the practice of visiting the gym and working on one's physical appearance through working out and training. A user posted "I go to the gym 5 days per week and eat a clean and balanced diet" (Post 79). The sentiment here is that despite these practices, this particular user is unable to derive joy or change his social status through gymmaxxing, and therefore it does not provide a sufficient distraction from considering suicide.

\section{Media and Entertainment}

Various media entertainment consumption such as anime and K-Pop (i.e., Korean popular music) provide potential distractions for five users. Some users $(n=3)$ described utilizing anime or K-pop as a cope, and one user stated, "It's been a good bad fun, even running kpop game didn't work" (Post 52) and again, this did not provide effective relief from suicidal ideation and other depressive symptoms. Two posts described media entertainment such as music, movies, or video games, as evidenced in the following quote: "It's nice living life watching movies playing games. But it's all just a fucking cope" (Post 28). Although recognizing that media entertainment can offer a coping mechanism, it is altogether not sustainable, particularly in the long-term.

\section{The Effects for Others}

A consideration for others in regard to an incel's decision to die by suicide was mentioned in many posts $(n=16)$. The effect of their suicide on their surrounding family members and friends was essential in dictating their subsequent action of completing suicide. The findings were further divided into positive concerns $(n=12)$ and negative concerns $(n=3)$. 


\section{Positive Concerns}

Positive concerns were those effects that considered the potential impact on family members and friends. For instance, one user stated, "I just hope my mom won't be devasted [sic] when she hears about me" (Post 86). Another user wrote about his brother, "I didn't want to hurt him with such terrible news. I didn't want him to blame himself, questioning if he could have changed things. I didn't want to hurt him" (Post 53). Most of the effects had positive undertones in that incels worried about the impact of their death on their family members. This consideration depicts the seriousness with which suicide was contemplated by incel users.

\section{Negative Concerns}

Alternatively, negative concerns were those that depicted the effect of suicide on family members and friends as overtly negative and pessimistic, as their suicide may serve as revenge or punishment for suicide survivors. These sentiments were shrouded in a carelessness towards the people in their life. One user wrote "I am not going to leave a [suicide] note because I could [sic] give a damn about my evil mother" (Post 15) and another posted, "I've maxxed out my credit card. Why? Because my sister is guarantor on the card and that dumb foid will be left to pay the debt" (Post 18). In this instance, the user planned to leave financial repercussions for his sister after his passing. Of note is the dehumanizing term, foid (short for femoid)—commonly used on incel forums-which can justify inhumane treatment and objectify and degrade women. (For further reading about such metaphors, in which women are food, animals, meat, body parts, objects, or containers, see Praźmo, 2020.) Although there were more positive concerns than negative, both experiences are valid circumstances that incels navigate to ultimately decide to die by suicide.

\section{The Afterlife}

Another theme that was present in the data is the mentioning of an afterlife in at least 25 percent of the posts $(n=20)$, in both explicit and implicit ways. The following quotes capture the ways in which incels alluded to an afterlife: "hope to see you all in a better place" (Post 19), "Hopefully I'll see you all again somewhere nicer than here" (Post 4) and "It's been a pleasure brocels. I'll see you on the other side" (Post 5). The afterlife here is not any one thing, but rather a culmination of ideas and experiences that reflect an understanding that after death there is something to look forward to. 


\section{Discussion}

The first theme we identified related to the method that the authors of the posts shared. While many used the term "rope" (either as a verb or noun) to describe the act of attempting suicide, 26 posts described clear methods including ingestion of lethal chemicals or pills, jumping, suffocation or hanging, and guns. The lack of explanation from the remaining posts leaves space for speculation and further inquiry; however, the types and rates of methods used are rather inconsistent with the existing literature about gender and methods of suicide, with the most common method being ingestion $(n=8)$. Relating back to Adjacic-Gross and colleagues' (2008) assertion that hanging is the most predominant method of suicide among men worldwide, we have to consider that perhaps those who used the term "rope" colloquially may have been using the term literally or that there are other gendered reasons for which these incels chose less common methods among men. We must also weigh the notion that many of the incels were not living in the United States, and guns may be less accessible in other parts of the world. While again, the 2020 incels.co survey may not be generalizable to incels of Reddit, only 38 percent of respondents indicated that they lived in North America (and there was no distinction between countries). More than 40 percent were from Europe, with the remaining responses being Central/South America, Asia, Africa, and Oceania.

The internet plays a significant role in the proliferation of both pro- and anti-suicide sentiments, many of which were present on the sub-Reddit, r/IncelGraveyard. An assessment of the internet's role in promoting said messages about suicide revealed the ways in which the internet was used for researching and purchasing means necessary to complete suicide. Furthermore, the internet houses a variety of online groups and communities, such as the sub-Reddit, r/IncelTears, which can have a detrimental impact on the mental health of vulnerable incels, particularly those with suicidal ideation. Consistent with research about social media and peer pressure to die by suicide (Lavis \& Winter, 2020), we found that the users utilized the internet in various ways to distract themselves from their problems, find support, or research ways to complete their suicide. Though this may not be peer pressure in the traditional sense, on incel forums and discussion boards, there is often explicit support and enthusiasm for suicide as a means to address individual incel problems and end suffering. The influence of the internet should not be underestimated in determining its role in the promotion of suicide.

In addition to understanding the impact that the internet has on incels' suicide ideations, it is vital to interrogate the role of online communities and themes about suicide occurring within these contexts. Considering the ability of informal supports to provide a mediating factor for attempting suicide, incels' reliance on their digital community is an important source of support. Our findings show the overwhelmingly positive experiences 
"Qualitative Criminology"

that incels had on various incel forums and sub-Reddits. These digital spaces are an important source of interrogation for the current study. By to providing support and companionship (through digital means) they likely would not otherwise have met in person, these spaces also offer a safe haven for incels who feel otherwise ostracized and marginalized by the larger society. The online community is a place where these men can express insecurities and failures with one another without fear of repercussions and judgment. Although we saw a few cases wherein incels had negative experiences on incel forums, the overwhelming experiences were positive, encouraging, and supportive, yet insufficient in addressing suicidal ideations. Though they may have felt a sense of belonging in the online groups, it did little to quell feelings of hopelessness and suicidality, contrary to the findings of Fisher et al. (2015).

Additionally, the current study explored how incel men navigated alternative methods-such as mental health treatment and distracting strategies like coping mechanisms - as a means of combating suicidal ideation. The inability to access mental health resources like therapists and medication due to feelings of anxiety, shame, and fear is consistent with research that identifies similar barriers to effective mental health treatment (Alonzo et al., 2017; Gibbons et al., 2010). Furthermore, those users who reported being able to access various mental health resources reported inadequate responses in alleviating their problems. In many countries, a lack of access to resources (or overwhelming pressure on the healthcare system) may hinder some incels' ability to receive the care that they need if they do, in fact, seek treatment. In one instance, a user reported being laughed at by the hospital security guards, and another was given seemingly ineffective support through the suicide prevention hotlines (Post 2 and Post 44 , respectively). These concerns are important to consider, especially when considering potential policy-level preventive tactics to combat suicide, particularly suicide of individuals from such exclusive and inaccessible communities like incels.

Further, the coping strategies shared by incels most commonly reflected the most negative or ineffective types of categories, such as distractions, avoidance, or disengagement. Many of the approaches they described in their suicide posts-such as video games and anime-fail to address the root concerns of their problems (compared to using a problem- or emotion-focused strategy), although they may be considered positive, as Fogarty et al. (2015) described finding enjoyable activities as a positive strategy.

Others used looksmaxxing or gymmaxxing to address the problems with their physical appearance, which can be classified as utilizing a problem-focused strategy. This may be categorized as using existing masculinity to exhibit hegemonic attributes and conform to the male gender expectations and norms (Spendelow \& Seidler, 2020). Even more concerning, Emslie et al. (2006) found that "the pressures of conforming to the 
standards of hegemonic masculinity could contribute to suicidal behaviour" (p. 2246). This is unique, in that generally (and correctly) view themselves as being a subordinated masculinity (Connell \& Messerschmidt, 2005), although in the context of incel forums, they may often use discursive practices to achieve and reiterate hegemonic masculinity (Daly \& Reed, 2021; Ging, 2019).

Moreover, the use of school or jobs to deflect from their problems and emotions may be considered a distraction (particularly as it is classified and defined in this study), but again are not problem- or emotion-focused. Instead, it seems that the authors of the posts have failed at using their jobs or schoolwork as alternative strategies to address their issues, and when the distraction failed, they remained stuck in a situation in which suicide seemed like the best option.

The majority of incels who considered the effects of their suicide on others were, for the most part, concerned about negative consequences and emotions for those they were leaving behind. Of the 16 incels who expressed concern for others (20 percent of the total sample), 75 percent were worried about the impact of their death on their family members. This is far greater than the 6.5 percent of notes from men in the Li et al. (2020) study that mentioned care for their family. Only three of the incels viewed their suicide and subsequent death as punishment or revenge for their suicide survivors. Both subthemes, demonstrate that some of the users considered and recognized that their death could have either positive or negative effects for their loved ones.

Finally, 25 percent of the posts referenced some type of afterlife either in vague or explicit ways. In the context of declining religious affiliations among younger generations (Pew Research Center, 2019), but increases in belief in the afterlife among millennials (Brean, 2018), this is far lower than the expected percentage. However, given that these are all suicide notes and the research findings that religiosity can be a protective factor (Gwim et al., 2020), it seems that the relatively low frequency of discussion of the afterlife is logical and somewhat expected. It is also important to consider that the afterlife can be an important element of suicidal ideation and often sustains the decision to die by suicide. A belief in some element of the afterlife may provide a sense of comfort and support, particularly for the members of this community.

\section{Limitations}

First and foremost, we recognize that the lack of descriptive information about the authors of these posts makes it difficult to generalize to other incels and the broader population who may be at risk for suicide and self-harm. We do note, though, that what we can learn from these posts-without any further descriptive statistics about those who posted 
them-can lead researchers, mental health practitioners, and others to better understand the frame of mind of those who claim to be incels and how they have framed their experiences, their attempts at self-improvement, and their concerns related to suicide.

Second, we cannot know if the authors of these posts did, in fact, die by suicide. Yet, regardless of outcomes, it is clear that these men experienced suicidal ideations, defined by Harmer et al. (2020) as "a broad term used to describe a range of contemplations, wishes, and preoccupations with death and suicide" (p. 1). Further, given the clear indications that many incels experience depression and other symptoms consistent with mental health conditions (ADL, 2020), it remains necessary to understand the interactive nature of suicidal ideations and other concerns. This is an important consideration in the implications for policy and treatment as "[t]herapists may be reluctant to treat patients with suicidal ideation because of the inherent risk of [hurting or killing themselves] ... [as] no clinician in academia or the community wants to manage the aftermath of a patient suicide" (Schneider et al., 2020, p. 500).

We further note that these posts as artifacts are not representative of a linear thought- or decision-making process toward suicide. Instead, they capture a single moment in time, thus limiting the explanatory power of any of these variables, but they also provide insight into the grievances, concerns, and experiences that may influence incels which can hopefully guide future research and policy.

Finally, there is notable concern that this list of incels who had considered and possibly attempted or completed suicide is not comprehensive. As one incel noted, "There are loads that aren't on the list [to be honest]. Plus, it's not updated too often ... [IncelGraveyard] only seems to focus on Reddit incels. Not any that are exclusively elsewhere that kill themselves" (Frail, 2020, personal communication). For methodological purposes, this article sampled the only known collection of incel suicide posts, but future research should examine other forums for similar evidence of suicidal ideations and posts from members, as there are likely many more cases of incel suicide that remain undocumented or unknown.

\section{Implications for Future Research and Policy}

This research study aimed to address an understudied aspect of incel experiences and perspectives. While it provided a foundation for the analysis of suicide notes, we hope that it can provide direction for future research to better understand factors that drive incel suicide. For qualitative research, most of the existing research examines the vitriolic rhetoric of incel forums and highlights the potential for large-scale incel violence, and thus, it is critical for researchers to explore the underlying issues that may lead to the suicide 
and self-harm that appear to be a more likely outcome for men who are incels. While there is limited quantitative research on incels (see Speckhard et al., 2021, for example), future studies should consider comprehensive, multi-variable measures of suicidality.

Even more, the discussion of failed coping strategies, suicide methods, and the social function of online groups should encourage academic inquiry and consideration from the mental health research communities. Continued attention about this aspect of inceldom, as well as research about the reasons or justification that incels provide for their suicide, is certainly warranted given the limitations of treatment options with those who struggle with suicidal ideation. Further, given the existing research about the link between suicidality and mass violence (Lankford, 2018; Peterson \& Densley, 2021), there is an urgent need to approach large-scale violence prevention with a suicide prevention lens.

Future research in criminology, criminal justice, and mental health research related to incels should consider including incels in the research to gain a more complete picture of the issues rather than simply relying on a single online post or online interactions to represent the whole of human behavior. We recognize that this can be difficult given that incels can be considered a hard-to-reach or hidden population (Shaghaghi et al., 2011) that may also be hostile toward researchers. If we aim to reduce and prevent instances of self-harm, suicide, and violence, researchers must create a trusting, positive, professional relationship with members of the incel community to engage in personcentered research and identify effective intervention efforts.

In addition to undertaking an informed approach for future research, it is imperative to discuss implications for policy and practice when it comes to incels and suicide prevention. To effectively address suicide prevention of a population like incels, the approach must be inclusive and specific to incels. Considering the unique perspectives and circumstances that incels face, they appear to experience increased social isolation, ostracism, and validated feelings of victimization, particularly by other online groups like $\mathrm{r} / \mathrm{IncelTears.} \mathrm{These} \mathrm{experiences} \mathrm{legitimate} \mathrm{feelings} \mathrm{of} \mathrm{depression} \mathrm{and} \mathrm{anxiety,} \mathrm{which} \mathrm{can}$ lead to or increase existing suicidal ideation (Katz et al., 2013; McClellan et al., 2021). Considering there is a documented relationship between cyberbullying and suicidal ideation (Patchin \& Hinduja, 2010; Van Geel et al., 2014), it is important to introduce effective policies to address the targeting of populations like incels. Although it is not enough to say that cyberbullying leads to attempting suicide, the significance of online groups bullying and vilifying incels cannot be understated.

Furthermore, as with other suicide prevention tactics, incels' suicide prevention must be addressed within a trauma-informed approach by law enforcement and medical 
professionals. As evidenced in one user's recalling of seeking mental health treatment, he shared that he was laughed at by the hospital guards (although there is no further context given in the note) which led to him leaving the hospital and contemplating suicide. This perceived mistreatment of vulnerable individuals' psychological well-being by practitioners undermines the efficacy of mental health programs. Understanding how difficult it is for vulnerable individuals to access such services in the first place, it is important to establish protocols that address the unique mental health concerns of incels.

\section{Conclusion}

The variety of themes presented in the incel suicide posts have provided new and unique insight into incel experiences, perceptions, and coping strategies before they ultimately decide to attempt suicide. This contributes to the existing literature about incels by using a new source of data to understand underlying issues. While many people may find online incel spaces to be hateful, toxic places, this research can potentially reframe incel issues and potential for violence to include issues of self-harm and suicide. By studying these critical aspects of experiences, we have the opportunity to expand upon existing incel research to better understand the complexity and the nuances of incel issues. Given the existing knowledge about suicide-homicide and extremism, there is no doubt that prevention efforts must also address the underlying issues of self-harm and suicide.

\section{References}

Anti-Defamation League (ADL). (2020). Online poll results provide new insights into incel community. https://www.adl.org/blog/online-poll-results-provide-new-insights-into-incelcommunity

Ajdacic-Gross, V., Weiss, M. G., Ring, M., Hepp, U., Bopp, M., Gutzwiller, F., \& Rossler, W. (2008). Methods of suicide: International suicide patterns derived from the WHO mortality database. Bulletin of the World Health Organization, 86, 736-732.

Alonzo, D., Moravec, C., \& Kaufman, B. (2017). Individuals at risk for suicide: Mental health clinicians' perspectives on barriers to and facilitators of treatment engagement. Crisis: The Journal of Crisis Intervention and Suicide Prevention, 38(3), 158-167.

Bachmann, S. (2018). Epidemiology of suicide and the psychiatric perspective. International Journal of Environmental Research and Public Health, 15(7), 1-23.

Biddle, L., Donovan, J., Hawton, K., Kapur, N., \& Gunnell, D. (2008). Suicide and the internet. BMJ, 336(7648), 800-802. 
Bjerkeset, O., Romundstad, P., \& Gunnell, D. (2008). Gender differences in the association of mixed anxiety and depression with suicide. The British Journal of Psychiatry, 192(6), 474-475.

Blair-West, G. W., Cantor, C. H., Mellsop, G. W., \& Eyeson-Annan, M. L. (1999). Lifetime suicide risk in major depression: Sex and age determinants. Journal of Affective Disorders, 55(2), 171-178.

Boslvert, N. (2020). Homicide at Toronto massage parlour was an act of incel terrorism, police say. CBC. https://www.cbc.ca/news/canada/toronto/incel-terrorism-massageparlour-1.5575689

Braun, V., \& Clarke, V. (2006). Using thematic analysis in psychology. Qualitative Research in Psychology, 3(2), 77-101.

Brean, J. (2018, March 29). Millennials are more likely to believe in an afterlife than are older generations. National Post. https://nationalpost.com/news/canada/millennials-doyou-believe-in-life-after-life

Carpenter, B., Bond, C., Tait, G., Wilson, M., \& White, K. (2016). Who leaves suicide notes? An exploration of victim characteristics and suicides in Queensland. Archives of Suicide Research, 20, 176-190.

Carver, C. S., \& Connor-Smith, J. (2010). Personality and coping. Annual Review of Psychology, 61, 679-704.

Chatfield, S. L. (2018). Considerations in qualitative research reporting: A guide for authors preparing articles for Sex Roles. Sex Roles: A Journal of Research, 79(3-4), 125135.

Check, J. V. P., Perlman, D., \& Malamuth, N. M. (1985). Loneliness and aggressive behaviour. Journal of Social and Personal Relationships, 2, 243-252.

Coimbra-Gomes, E., \& Motschenbacher, H. (2019). Language, normativity, and sexual orientation obsessive-compulsive disorder (SO-OCD): A corpus-assisted discourse analysis. Language in Society, 48(4), 565-584.

Connell, R. W., \& Messerschmidt, J. W. (2005). Hegemonic masculinity: Rethinking the concept. Gender \& Society, 19(6), 829-859. 
Costello, W. (2020, August 6). Step your dick up: Why incels deserve better advice. Medium. $\quad$ https://williamcostello.medium.com/step-your-dick-up-why-incels-deservebetter-advice-307879d7c97b

Daly, S. E., \& Reed, S. R. (2021). "I think society hates us": A qualitative thematic analysis of interviews with incels. Sex Roles: A Journal of Research.

Deane, F. P., Wilson, C. J., \& Ciarrochi, J. (2001). Suicidal ideation and help-negation: Not just hopelessness or prior help. Journal of Clinical Psychology, 57(7), 901-914.

Donnelly, D., Burgess, E., Anderson, S., Davis, R., \& Dillard, J. (2001). Involuntary celibacy: A life course analysis. Journal of Sex Research, 38(2), 159-169.

Dynel, M. (2020). Vigilante disparaging humour at r/IncelTears: Humour as critique of incel ideology. Language \& Communication, 74, 1-14.

Emslie, C., Ridge, D., Ziebland, S., \& Hunt, K. (2006). Men's accounts of depression: Reconstructing or resisting hegemonic masculinity? Social Science \& Medicine, 62(9), 2246-2257.

Farrell, T., Fernandez, M., Novotny, J., \& Alani, H. (2019). Exploring misogyny across the Manosphere in Reddit. WebSci '19: Proceedings of the $10^{\text {th }}$ ACM Conference on Web Science, p. 87-96.

Felsten, G. (1998). Gender and coping: Use of distinct strategies and associations with stress and depression. Anxiety, Stress \& Coping: An International Journal, 11(4), 289309.

Fisher, L.B., Overholser, J.C., Ridley, J., Braden, A., \& Rosoff, C. (2015). From the outside looking in: Sense of belonging, depression, and suicide risk. Psychiatry, 78, 2941.

Fogarty, A. S., Proudfoot, J., Whittle, E. L., Player, M. J., Christensen, H., Hadzi-Pavlovic, D., \& Wilhelm, K. (2015). Men's use of positive strategies for preventing and managing depression: A qualitative investigation. Journal of Affective Disorders, 188, 179-187.

Folkman, S. (1997). Positive psychological states and coping with severe stress. Social Science \& Medicine, 45(8), 1207-1221. 
Fredborg, L. (2013). Extract comments to new document. DocTools. https://www.thedoctools.com/word-macros-tips/word-macros/extract-comments-to-newdocument/

Furqan, Z., Sinyor, M., Schaffer, A., Kurdyak, P., \& Zaheer, J. (2019). "I can't crack the code": What suicide notes teach us about experiences with mental illness and mental health care. Canadian Journal of Psychiatry, 64(2), 98-106.

Galasiński, D. (2017). Discourses of men's suicide notes: A qualitative analysis. Bloomsbury.

Gibbons, C. J., Wiltsey-Stirman, S., Brown, G. K., \& Beck, A. T. (2010). Engagement and retention of suicide attempters in clinical research. Crisis, 31, 62-68.

Ging, D. (2019). Alphas, betas, and incels: Theorizing the masculinities of the Manosphere: Men and Masculinities, 22(4), 638-657.

Gilmartin, B. G. (1987). Peer group antecedents of severe love-shyness in males. Journal of Personality, 55(3), 467-489.

Glace, A. M., Dover, T. L., \& Zatkin, J. G. (2021). Taking the black pill: An empirical analysis of the "Incel". Psychology of Men \& Masculinities, 22(2), 288-297.

Gunn, J. F. (2014). Fatal loneliness: The role of loneliness in men's suicide. In D. Lester, J. F. Gunn III, \& P. Quinnett (Eds.), Suicide in men: How men differ from women in expressing their distress (pp. 34-43). Springfield.

Gwim, S., Branscum, P., Taylor, E. L., Cheney, M., Maness, S. B., Frey, M. \& Zhang, Y. (2020) Associations between suicide behaviors and religiosity in young adults. American Journal of Health Studies, 35(4), 257-269.

Harmer, B., Lee, S., Duong, T. H., \& Saadabadi, A. (2020). Suicidal ideation. In: StatPearls [Internet]. https://pubmed.ncbi.nlm.nih.gov/33351435/

Harvard Medical School. (2019, May 29). Left behind after suicide. https://www.health.harvard.edu/mind-and-mood/left-behind-after-suicide

Hedegaard, H., Curtin, S. C., \& Warner, M. (2020, April). Increase in suicide mortality in the United States, 1999-2018. Centers for Disease Control and Prevention. https://www.cdc.gov/nchs/products/databriefs/db362.htm 
Heritage, F., \& Koller, V. (2020). Incels, in-groups, and ideologies: The representation of gendered social actors in a sexuality-based online community. Journal of Language and Sexuality, 9(2), 152-178.

Hoffman, B., \& Ware, J. (2020). Assessing the threat of incel violence. Studies in Conflict \& Terrorism, 43(7), 565-587.

Hom, M. A, Stanley, I. H, Joiner Jr., T. E. (2015). Evaluating factors and interventions that influence help-seeking and mental health service utilization among suicidal individuals: A review of the literature. Clinical Psychology Review, 40, 28-39.

Illner, N., Gerth, J., Pfeiffer, R., Bruns, T., \& Wolf, G. (2009). "Nearly a stairway to heaven": Severe dichromate intoxication in a young man. Clinical Nephrology, 71(3), 338341.

Katz, I. R., Kemp, J. E., Blow, F. C., McCarthy, J. F., \& Bossarte, R. M. (2013). Changes in suicide rates and in mental health staffing in the veterans' health administration, 20052009. Psychiatric Services, 64(7), 620-625.

Koenig, H. (2008). Medicine, religion, and health: Where science and spirituality meet. Templeton Foundation Press.

Kołaciński, Z., Kostrzewsii, P., Kruszewska, S., Razniewska, G., \& Mielczarska, J. (1999). Acute potassium dichromate poisoning: A toxicokinetic case study. Journal of Toxicology and Clinical Toxicology, 37(6), 785-791.

Krysinska, K. (2014). Men and suicide: An overview. In D. Lester, J.F. Gunn III, \& P. Quinnett (Eds.). Suicide in Men: How men differ from women in expressing their distress. Charles C Thomas Publisher Ltd.

Lankford, A. (2013). The myth of martyrdom: What really drives suicide bombers, rampage shooters, and other self-destructive killers. Palgrave Macmillan.

Lankford, A. (2016). Fame-seeking rampage shooters: Initial findings and empirical predictions. Aggression \& Violent Behavior, 27, 122-129.

Lankford, A. (2018). Identifying potential mass shooters and suicide terrorists with warning signs of suicide, perceived victimization, and desires for attention or fame. Journal of Personality Assessment, 100(5), 471-482. 
Lavis, A., \& Winter, R. (2020). \#Online harms or benefits? An ethnographic analysis of the positives and negatives of peer-support around self-harm on social media. The Journal of Child Psychiatry and Psychology, 61(8), 842-854.

Li, F., Lu, X., \& Yip, P. S. F. (2020). A study of the characteristics of suicide notes in China. Crisis, 41(1), 32-38.

Liu, X., Huang, J., Yu, N., Li, Q., \& Zhu, T. (2020). Mediation effect of suicide-related social media use behaviors on the association between suicidal ideation and suicide attempt: Cross-sectional questionnaire study. Journal of Medical Internet Research, 22(4), 316-328.

Maxwell, D., Robinson, S. R., Williams, J. R., \& Keaton, C. (2020). "A short story of a lonely guy": A qualitative thematic analysis of involuntary celibacy using Reddit. Sexuality \& Culture, 24, 1852-1874.

McAlister, A. M., Lee, D. M., Ehlert, K. M., Kajfez, R. L., Faber, C. J., \& Kennedy, M. S. (2017). Qualitative coding: An approach to assess inter-rater reliability. American Society for Engineering Education.

McClellan, C., Ali, M., \& Mutter, R. (2021). Impact of mental health treatment on suicide attempts. The Journal of Behavioral Health Services \& Research, 48(1), 4-14.

McCorkel, J., \& Meyers, K. (2003). What difference does difference make? Position and privilege in the field. Qualitative Sociology, 26(2), 199-231.

Menzie, L. (2020). Stacys, Beckys, and Chads: The construction of femininity and hegemonic masculinity within incel rhetoric. Psychology \& Sexuality. Advance Online Publication.

Miles, M. B., \& Huberman, A. M. (1994) Qualitative data analysis: An expanded sourcebook (2nd ed.). Sage Publications, Inc.

Mok, K., Jorm, A.F., \& Prikis, J. (2016). Who goes online for suicide-related reasons? A comparison of suicidal people who use the internet for suicide-related reasons and those who do not. Crisis: The Journal of Crisis Intervention and Suicide Prevention, 37(2), 112120.

Moreira-Almedia, A., Lotufo Neto, F., \& Koenig, H. G. (2006). Religiousness and mental health: A review. Journal of the Brazilian Psychiatric Association, 28, 242-250. 
Naples, N. A. (2003). Feminism and method: Ethnography, discourse analysis, and activist research. Routledge.

Neumeister, L (2021, April 14). 'Incel' teen held without bail on federal bomb threat charge. Associated Press. https://apnews.com/article/new-york-bomb-threats-arrestsmanhattan-2080251d9390401f387cfff16f6ef6bd

O'Malley, R. L., Holt, K., \& Holt, T. J. (2020). An exploration of the involuntary celibate (incel) subculture. Journal of Interpersonal Violence.

Olson, L. M., Wahab, S., Thompson, C. W., \& Durrant, L. (2011). Suicide notes among Native Americans, Hispanics, and Anglos. Qualitative Health Research, 21(11), 14841494.

Papadamou, K., Zannettou, S., Blackburn, J., De Cristofaro, E., Stringhini, G., \& Sirivianos, M. (2020). Understanding the incel community on YouTube. https://arxiv.org/pdf.2001.08293.pdf

Patchin, J. W., \& Hinduja, S. (2006). Bullies move beyond the schoolyard: A preliminary look at cyberbullying. Youth Violence and Juvenile Justice, 4(2), 148-169.

Pestian, J. P., Matykiewicz, P., Linn-Gust, M. \& South, B. (2012). Sentiment analysis of suicide notes: A shared task. Biomedical Informatics Insights, 5(Suppl 1), 3-16.

Peterson, J., \& Densley, J. (2021, March 27). Why mass shootings stopped in 2020 - and why they are roaring back now. Wisconsin State Journal. https://madison.com/wsj/opinion/column/jillian-peterson-and-james-densley-why-massshootings-stopped-in-2020-and-why-they-are/article 087e40a0-0451-5ce4-9b5c130e87ca9341.html

Pew Research Center. (2019, October 17). In U.S., decline of Christianity continues at rapid pace. Religion \& Public Life. https://www. pewforum.org/2019/10/17/in-u-s-declineof-christianity-continues-at-rapid-pacel

Porter, C. (2021, March 3). Toronto van attacker found guilty in city's worst mass killing. The New York Times. https://www.nytimes.com/2021/03/03/world/canada/toronto-vanalek-minassian.html

Praźmo, E. (2021). Foids are worse than animals. A cognitive linguistics analysis of dehumanizing metaphors in online discourse. Topics in Linguistics, 21(2), 16-27. 
Rasmussen, M. L., Haavind, H., \& Dieserud, G. (2018). Young men, masculinities, and suicide. Archives of Suicide Research, 22, 327-343.

Recupero, P.R., Harms, S.E., \& Noble, J.M. (2008). Googling suicide: Surfing for suicide information on the internet. The Journal of Clinical Psychiatry, 69(6), 878-888.

Richardson, C., Robb, K. A., O'Connor, R. C. (2021). A systematic review of suicidal behaviour in men: A narrative synthesis of risk factors. Social Science \& Medicine.

rxlist.com (2021, April 23). Pentothal (Thiopental Sodium). https://www.rxlist.com/pentothal-drug.htm

Scaptura, M. N., \& Boyle, K. M. (2020). Masculinity threat, "incel" traits, and violent fantasies among heterosexual men in the United States. Feminist Criminology, 15(3), 278-298.

Schneider, R. A., Chen, S. Y., Lungu, A., \& Grasso, J. R. (2020). Treating suicidal ideation in the context of depression. BMC Psychiatry, 20, 497-501.

Serina, J. (2015, February 19). Elliot Rodger meticulously planned the Isla Vista rampage, report says. Los Angeles Times. https://www.latimes.com/local/lanow/la-me-In-santabarbara-isla-vista-rampage-investigation-20150219-story.html

Shaghaghi, A., Bhopal, R. S., Sheikh, A. (2011). Approaches to recruiting 'hard-to-reach' populations into research: A review of the literature. Health Promotion Perspective, 1(2), 86-94.

Smędra, A., Szustowski, S., Jurczyk, A. P., Klemm, J., Szram, S., \& Berent J. (2015). Suicidal asphyxiation by using helium: Two case reports. Archives of Forensic Medicine and Criminology, 65(1):37-46.

Speckhard, A., Ellenberg, M., Morton, J., \& Ash, A. (2021). Involuntary celibates' experiences of and grievance over sexual exclusion and the potential threat of violence among those active on an incel forum. Journal of Strategic Security, 14(2), 89-21.

Spendelow, J. S., \& Seidler, Z. E. (2020). Men's self-initiated coping strategies across medical, psychological, and psychosocial issues: A systematic review. Psychology of Men \& Masculinities, 21(1), 106-123. 
Texas Department of Public Safety. (2020). Texas domestic terrorism threat assessment. https://www.dps.texas.gov/sites/default/files/documents/director staff/media and com munications/2020/txterrorthreatassessment.pdf

Tranchese, A., \& Sugiura, L. (2021). "I don't hate all women, just those stuck-up bitches": How incels and mainstream pornography speak the same extreme language of misogyny. Violence Against Women, 27(14), 2709-2734.

Tsirigotis, K., Gruszczynski, W., \& Tsirigotis, M. (2011). Gender differentiation in methods of suicide attempts. Medical Science Monitor, 7(8), PH65-PH70.

Van Geel, M., Vedder, P., \& Tanilon, J. (2014). Relationship between peer victimization, cyberbullying, and suicide in children and adolescents: A meta-analysis. JAMA Pediatrics, 168(5), 435-442.

Zhang, J., Lester, D., Haines, J., Williams, C. L., Zhou, R., Qi, Q., Li, T., Liu, L. \& Ma, W. (2020). Identifying psychological strains in suicide notes. Journal of Death and Dying, 82(1), 120-127.

\section{Contributors}

Sarah E. Daly is an assistant professor of criminology, law, and society at Saint Vincent College in Latrobe, Pennsylvania. Her primary areas of research include inceldom, mass violence, and gender, and she recently published findings from her interviews with incels in Sex Roles. She is the author of Everyday School Violence: An Educator's Guide to Safer Schools and the editor of two volumes: Assessing and Averting Mass Violence and Theories of Crime through Popular Culture. Dr. Daly is also the co-founder and co-editor of the Journal of Mass Violence Research, and she can be found on Twitter @sarahdalycj.

Albina Laskovtsov is a doctoral student at the University of South Carolina working on a $\mathrm{PhD}$ in Criminology. She received a bachelor's degree in Criminology and Criminal Justice from Morehead State University and a master's degree in Criminology and Criminal Justice from Eastern Kentucky University. She specializes in violence against women, violence in cyberspace, and victimization.

\section{Acknowledgment}

The authors are grateful to those colleagues and friends who reviewed this paper and offered suggestions. 